\title{
Narrative review - how to access nodules: role of new technology including navi- and robo-bronchoscopy
}

\author{
Alexander Gregor^, Tsukasa Ishiwata^, Terunaga Inage^, Kazuhiro Yasufuku^ \\ Division of Thoracic Surgery, Toronto General Hospital, University Health Network, University of Toronto, Toronto, Ontario, Canada \\ Contributions: (I) Conception and design: All authors; (II) Administrative support: K Yasufuku; (III) Provision of study materials or patients: None; \\ (IV) Collection and assembly of data: All authors; (V) Data analysis and interpretation: All authors; (VI) Manuscript writing: All authors; (VII) Final \\ approval of manuscript: All authors. \\ Correspondence to: Kazuhiro Yasufuku, MD, PhD, FRCSC. 200 Elizabeth St, EN 9N-957, Toronto, Ontario, M5G 2C4, Canada. \\ Email: kazuhiro.yasufuku@uhn.ca.
}

\begin{abstract}
Implementation of lung cancer screening is expected to increase the detection of lung nodules, many of which will require intervention for diagnosis or management. Transbronchial approaches have generally shown a favourable risk profile relative to transthoracic techniques. Bronchoscopic interventions are therefore particularly attractive in comorbid and frail patient populations. Fundamental to any biopsy or local therapy is accurate device delivery. However, transbronchial techniques must work within the constraints of bronchial anatomy, which may not inherently lend itself to easy access of suspicious nodules. A variety of technologies have been developed to improve the clinicians' ability to reliably access nodules via a transbronchial approach. The MEDLINE database was searched from 2000 through June 2020 on the topic of navigation bronchoscopy and associated technologies. This article will review several technology classes, including intraoperative imaging that provides real-time anatomic information (computed tomography fluoroscopy, cone-beam computed tomography, augmented fluoroscopy), navigation systems that facilitate planning/selection of bronchial tree pathways [virtual bronchoscopic navigation (VBN), electromagnetic navigation bronchoscopy (ENB)], evolutions in bronchoscope design that improve function within the peripheral lung [ultrathin bronchoscopy (UTB), robotic bronchoscopy], and new platforms that permit access beyond the bronchial wall (bronchoscopic transparenchymal nodule access, transbronchial access tool). Familiarity with the strengths and limitations of these technologies are critical for their effective use.
\end{abstract}

Keywords: Navigation bronchoscopy; lung nodule; robotic bronchoscopy

Received: 24 June 2020; Accepted: 19 February 2021; Published: 25 November 2022.

doi: $10.21037 /$ ccts-20-122

View this article at: http://dx.doi.org/10.21037/ccts-20-122

\section{Introduction}

Results of the National Lung Screening Trial (NLST) and Dutch-Belgian lung-cancer screening trial (NELSON) demonstrated reduced lung cancer mortality with implementation of computed tomography (CT)-based screening $(1,2)$. This was achieved at least in part through a shift towards early stage diagnosis $(1,2)$. However, most positive scans were in fact false positives, and although there are several strategies for risk-stratifying pulmonary nodules, tissue diagnosis remains critical $(1,2)$. The population has at the same time become older and more comorbid, altering the risk-benefit calculation when considering standard

^ ORCID: Alexander Gregor, 0000-0001-7365-6084; Tsukasa Ishiwata, 0000-0003-2369-2698; Terunaga Inage, 0000-0001-5585-1601; Kazuhiro Yasufuku, 0000-0002-3053-4156. 
Table 1 Summary of technologies enabling nodule access

\begin{tabular}{|c|c|c|}
\hline Technology & Overview & Example commercial system (manufacturer) \\
\hline Computed tomography fluoroscopy & $\begin{array}{l}\text { Intraoperative cross-sectional images albeit } \\
\text { narrow field-of-view }\end{array}$ & \\
\hline Cone-beam computed tomography & $\begin{array}{l}\text { Intraoperative cross-sectional images, } \\
\text { fluoroscopy-capable }\end{array}$ & \\
\hline \multicolumn{3}{|l|}{ Navigation systems } \\
\hline Virtual bronchoscopic navigation & $\begin{array}{l}\text { Reconstruction of CT data to simulate } \\
\text { endoscopic view/path }\end{array}$ & $\begin{array}{l}\text { Bf-NAVI/DirectPath (Olympus), LungPoint } \\
\text { (Broncus) }\end{array}$ \\
\hline Ultrathin bronchoscopy & $\begin{array}{l}\text { Small diameter, highly maneuverable } \\
\text { bronchoscope }\end{array}$ & MP-190F/290F (Olympus) \\
\hline Robotic bronchoscopy & $\begin{array}{l}\text { Highly maneuverable, stable platform with } \\
\text { integrated navigation }\end{array}$ & Monarch (Auris), Ion (Intuitive) \\
\hline Transparenchymal access & $\begin{array}{l}\text { Puncture-and-dilating kit to access nodules } \\
\text { beyond the bronchial tree }\end{array}$ & $\begin{array}{l}\text { Archimedes (Broncus), CrossCountry } \\
\text { (Medtronic) }\end{array}$ \\
\hline
\end{tabular}

CT, computed tomography.

approaches for biopsy, localization, and therapy (3). CTguided transthoracic biopsy, the current gold-standard, has a pooled diagnostic yield of $93 \%$ but a pneumothorax rate of $26 \%$ (4). Surgery may not be feasible due to comorbidities or patient preference.

Transbronchial approaches have generally shown a lower complication rate than transthoracic approaches, with a pneumothorax rate as low as $1.5 \%$ (5). This has made it an appealing alternative approach for biopsy, localization, and therapy. In the context of biopsy, conventional bronchoscopy is generally effective for central lesions (88\% sensitivity) (6). However, this falls precipitously for peripheral lesions $(34 \%$ and $66 \%$ sensitivity for $<2$ and $>2 \mathrm{~cm}$ diameter lesions, respectively) (6). This diagnostic performance portends poorly for other transbronchial procedures; accurate delivery of biopsy forceps relies on the same principles as any other instrument. In this context, diagnostic yield can function as an effective surrogate for nodule access, though they are not necessarily equivalent. Nodule access (i.e., the ability to bring a bronchoscope or instrument into the region of a target) is necessary, but not sufficient, for successful biopsy. We direct readers to Dr.
Tsukada's review in this series for a more detailed discussion on considerations for transbronchial biopsy, including endobronchial ultrasound (EBUS) (7).

To address the challenge of nodule access, a variety of new technologies have been developed (Table 1). These technologies are frequently complementary, and many studies often evaluate several of them simultaneously. It is critical that bronchoscopists be familiar with the strengths and limitations of these individual components to develop a flexible and effective nodule access strategy.

\section{Methods}

For this review, we searched the MEDLINE database for human clinical studies published in English from 2000 to June 2020 and present the following article in accordance with the Narrative Review reporting checklist (available at https://ccts. amegroups.com/article/view/10.21037/ccts-20-122/rc).

\section{Intraoperative imaging}

Conventional two-dimensional (2D) fluoroscopy has two 
limitations. First, nodules may not be fluoroscopically visible. Second, fluoroscopy may be falsely reassuring if the bronchoscope/instrument is overlapping the target in a plane perpendicular to the fluoroscopic view (8). Advanced intraoperative imaging can facilitate nodule access by confirming instrument position relative to the nodule and, if positioning is inaccurate, informing selection of an alternate route.

\section{Computed tomography fluoroscopy}

Modern multidetector CT scanners can be employed for CT fluoroscopy. By positioning the patient such that the target nodule is centered within the scanner bore, the scanner can be intermittently activated to rapidly acquire real-time intraoperative cross-sectional images (9). This technique has the greatest utility for small lesions, which are likely fluoroscopically invisible. A retrospective study comparing multidetector CT fluoroscopy $(n=82)$ with 2D fluoroscopy $(n=78)$ found the diagnostic yield was significantly greater for small lesions $(42.9 \%$ versus $7.7 \%$ in lesions $<10 \mathrm{~mm}, 54.2 \%$ versus $20 \%$ in lesions $11-15 \mathrm{~mm}$ ), but unchanged for larger lesions (10). Although a later randomized trial found no advantage for CT fluoroscopy, this study did not stratify by nodule size and the lesions were relatively large in both groups (mean 46 and $37 \mathrm{~mm}$, respectively) (11).

\section{Cone-beam computed tomography}

It is generally impractical to install a multidetector CT scanner into an operating room/endoscopy suite due to its size and configuration. Cone-beam CT (CBCT) generates cross-sectional images through rotation of a $\mathrm{C}$-arm, which can be more easily adjusted and worked around during normal procedures. These systems have formed key part of so-called 'hybrid operating rooms' that integrate advanced imaging systems in procedure areas (12). A pilot study reported on 20 patients who underwent CBCT after failed transbronchial biopsy with conventional fluoroscopy and radial probe endobronchial ultrasound (RP-EBUS) (13). Addition of CBCT improved navigation success (i.e., visualization by RP-EBUS) from $50 \%$ to $75 \%(\mathrm{P}=0.02)$, and diagnostic yield from $50 \%$ to $70 \%(\mathrm{P}=0.04)$ (13).

\section{Augmented fuoroscopy}

Augmented fluoroscopy is the enhancement of standard 2D fluoroscopic images through the overlay of additional data, such as segmentation data from a corresponding CT scan. As CBCT can acquire both cross-sectional and 2D fluoroscopic images, CBCT segmentation data (e.g., nodule) can be overlaid onto the $2 \mathrm{D}$ fluoroscopic image, provided the patient is not moved (14). The augmentation data automatically compensates for changes in C-arm position (15). Pritchett et al. evaluated this technique in conjunction with electromagnetic navigation bronchoscopy (ENB) in 75 patients (15). Although lesions were small (median $16 \mathrm{~mm}$ ) and often fluoroscopically invisible (49\%), the overall diagnostic yield was $83.7 \%$ (15). This compares favourably with ENB experience (see section $E N B)$. However, the average number of CBCT scans per patient was 1.5 , making the value of augmented fluoroscopy alone unclear (15).

A potential upcoming platform is LungVision (Body Vision Medical Ltd., Israel), an FDA-approved system that can register preoperative CT data to standard intraoperative fluoroscopy using fluoroscopic tomosynthesis, a radiopaque bead-filled registration board, and artificial intelligence (16). Included software can generate a navigation plan that is overlaid onto the real-time fluoroscopy (17). A multicentre trial of 200 patients (average nodule size $17 \mathrm{~mm}$ ) was described in an abstract, reporting $86 \%$ navigation success (CBCT or RP-EBUS confirmation) and $78 \%$ diagnostic yield (17). Formal publication of the trial results is pending.

\section{Navigation systems}

As intraoperative imaging becomes more advanced (see section Augmented fluoroscopy), the distinction between imaging and navigation systems becomes somewhat arbitrary. At their core, navigation systems process preoperative CT images to generate a navigation route to a target. However, unlike augmented fluoroscopy, which collapses three-dimensional (3D) information into a $2 \mathrm{D}$ projection, navigation systems generally retain and display $3 \mathrm{D}$ information throughout the procedure.

\section{Computed tomography-body divergence}

The registration of high-quality preoperative images is foundational to all navigation systems. However, the lag between scan acquisition and bronchoscopy can lead to discrepancy between CT and 'current' patient anatomy, referred to as CT-body divergence. The first challenge is practical: the procedure may no longer be necessary. One study found same-day CT scan resulted in cancellation of 
approximately $7 \%$ of procedures due to nodule regression (18). The second challenge is nodule displacement from breathing. A retrospective study comparing inspiratory and expiratory phase CT scans in 46 patients found nodule displacement was on average $17.6 \mathrm{~mm}$, but varied from $10.6 \mathrm{~mm}$ in the left upper lobe to $25.3 \mathrm{~mm}$ in the right lower lobe (19). The displacement vector was also complex, occurring primarily along the anteroposterior and craniocaudal planes (19). A third challenge is nodule displacement by bronchoscope wedging. A study in pigs with positional sensors placed in the bronchial tree found that although bronchoscope movement in the central airways caused minimal disruption, wedging the bronchoscope in a target segment displaced the associated sensor on average by $8.2 \mathrm{~mm}$ (20). A final challenge is that intraoperative changes, such as atelectasis, may alter nodule position. CT-body divergence establishes a clear role for complementary real-time imaging (e.g., RP-EBUS, CT, fluoroscopy) during navigation bronchoscopy.

\section{Virtual bronchoscopic navigation (VBN)}

$\mathrm{VBN}$ is the $3 \mathrm{D}$ reconstruction of the airways from CT data to simulate the expected endoscopic view (21). Images of the virtual lumen can then be directly compared with findings during bronchoscopy. VBN can be used for simulation (i.e., preoperative rehearsal) or navigation (i.e., intraoperative cross-reference with bronchoscopy). The two approaches were compared in a retrospective study using ultrathin bronchoscopy (UTB) and CT fluoroscopy (22). Diagnostic sensitivities were similar ( $63 \%$ versus $70 \%, \mathrm{P}=0.48$ ), but simulation was associated with longer time to first biopsy (13 versus $8.5 \mathrm{~min}, \mathrm{P}<0.05$ ) and longer total exam time (29.3 versus 24.5 min, $\mathrm{P}<0.05$ ) (22). Most contemporary uses of VBN employ the navigation approach.

As VBN is a form of CT reconstruction, it is feasible to perform VBN with generic CT workstation software, though more manual work is generally required (23-25). The bulk of VBN data focuses on two systems purpose-built for VBN: DirectPath/Bf-NAVI (Olympus Corporation, Japan) and LungPoint (Broncus Medical Inc., USA). These systems have advanced planning features, such as assisted target segmentation and automated path generation, and user interfaces better designed for intraoperative use. DirectPath/Bf-NAVI includes a viewer that displays the virtual lumen and highlighted navigation route; however, the reconstruction must be manually advanced/withdrawn/ rotated as the procedure progresses (26). LungPoint includes image recognition capabilities for automatic alignment of VBN images with the bronchoscopic view (27). When synchronized, target position and the navigation route can be overlaid onto the endoscopic video (27).

VBN is often combined with other modalities. Several trials support that VBN allows faster navigation to nodules when combined with RP-EBUS. However, the benefit for navigation success or diagnostic yield is less clear. Oshige et al. prospectively compared transbronchial nodule biopsy using RP-EBUS plus guide sheath (GS) with $(\mathrm{n}=57)$ or without $(\mathrm{n}=55)$ Bf-NAVI (28). Diagnostic yield was not significantly different $(84.2 \%$ versus $80 \%$ ), but time to first biopsy was shorter with VBN (5.5 versus $9.3 \mathrm{~min}, \mathrm{P}=0.0004$ ) (28). Bo et al. randomized 1,010 patients to unassisted transbronchial biopsy, RP-EBUS/GS-assisted biopsy, or RPEBUS/GS plus VBN-assisted biopsy for nodules $8-30 \mathrm{~mm}$ in diameter (29). Diagnostic yield was lowest without guidance (41.2\%, $\mathrm{P}<0.01)$, but no advantage was seen adding VBN to RP-EBUS/GS (74.3\% and $72.3 \%$, respectively), regardless of nodule size (29). RP-EBUS localization rates did not improve with $\mathrm{VBN}$, although the time to localize was shorter (7.96 versus $11.92 \mathrm{~min}, \mathrm{P}<0.01$ ) (29).

If $\mathrm{VBN}$ is to have any benefit on nodule access, it is most likely for small nodules. Ishida et al. randomized 199 patients with nodules $\leq 30 \mathrm{~mm}$ to Bf-NAVI-assisted or unassisted transbronchial biopsy; a thin bronchoscope (4.0 $\mathrm{mm}$ outer diameter) and RP-EBUS were used in both groups (30). Diagnostic yield was significantly improved with VBN (80.4\% versus $67 \%, \mathrm{P}=0.032)$, which appeared to be driven by nodules $<20 \mathrm{~mm}(75.9 \%$ versus $59.3 \%$ for $<20 \mathrm{~mm}, \mathrm{P}=0.056 ; 87.8 \%$ versus $80.6 \%$ for $20-30 \mathrm{~mm}$, $\mathrm{P}=0.382$ ) (30). RP-EBUS visualization improved with VBN (92.9\% versus $81.1 \%, \mathrm{P}=0.014$ ) (30). Time to first biopsy was reduced (8.1 versus $9.8 \mathrm{~min}, \mathrm{P}=0.045$ ) (30). $\mathrm{Xu}$ et al. randomized 115 patients to DirectPath-assisted or unassisted transbronchial biopsy using RP-EBUS, and found on subgroup analysis that VBN improved diagnostic yield in nodules $<20 \mathrm{~mm}$ ( $80 \%$ versus $53.6 \%, \mathrm{P}=0.041)$ (31). Time to RP-EBUS localization was shorter with VBN (5.7 versus $8.6 \mathrm{~min}, \mathrm{P}=0.015)(31)$.

Studies without RP-EBUS do not unmask additional VBN advantages. Asano et al. randomized 334 patients with nodules $\leq 30 \mathrm{~mm}$ to Bf-NAVI-assisted or unassisted transbronchial biopsy using UTB (2.8 mm outer diameter) (32). There was no significant difference in diagnostic yield (67.1\% versus $59.9 \%$, $\mathrm{P}=0.173)$, although a benefit was seen for peripheral lesions (64.7\% versus $52.1 \%, \mathrm{P}=0.047$ ) (32). Time to first biopsy was shorter with VBN, although the effect size was smaller than in other studies (6.4 versus $6.8 \mathrm{~min}, \mathrm{P}=0.021)$ (32). A similar 
matched case-control study compared unguided UTB $(\mathrm{n}=110)$ with LungPoint-assisted UTB (n=55) (33). Diagnostic yield was not significantly improved with $\mathrm{VBN}$ (47\% versus $40 \%$, $\mathrm{P}=0.354)$, though a trend to significance was noted for nodules $\leq 20 \mathrm{~mm}$ (42\% versus 24\%, $\mathrm{P}=0.069$ ) (33).

An interesting question is whether $\mathrm{VBN}$, which is reconstructed preoperative cross-sectional imaging, has any value over real-time cross-sectional imaging. Kato et al. prospectively compared 100 patients undergoing transbronchial CT fluoroscopy-guided biopsy with or without LungPoint-assistance (34). All nodules were $<20 \mathrm{~mm}$, peripheral, and had a bronchus sign (34). Surprisingly, diagnostic yield was higher with LungPoint (84\% versus $58 \%$, $\mathrm{P}=0.013$ ) (34). One possible explanation is that more biopsies were taken with $\mathrm{VBN}$ (3.54 versus $2.98, \mathrm{P}=0.01$ ), given the shortened time to first biopsy (15 versus $19 \mathrm{~min}, \mathrm{P}=0.008$ ) (34). However, it is also likely that interpretation of $\mathrm{VBN}$ is more intuitive than interpretation of axial CT imaging.

In summary, VBN's most consistent benefit is reduced time-to-target, providing more time for the procedure proper (e.g., biopsy). Although data is less consistent, VBN likely has benefit for small peripheral nodules, whose access route may be more difficult to plan without assistance. Leveraging this advantage requires use of technologies suitable for peripheral access (e.g., RP-EBUS, UTB). An advantage of $\mathrm{VBN}$ is its accessibility. Once installed there is no per-procedure cost, and interpretation of the navigation guidance is intuitive. An obvious limitation is the reliance on the endoscopic view; the utility of $\mathrm{VBN}$ diminishes if the endoscopic view is obscured (e.g., bleeding, mucus plugging) or if the bronchoscope cannot be physically advanced along the planned route.

\section{$E N B$}

When an electromagnetic sensor moves through an electromagnetic field, changes in voltage can be used to calculate its $3 \mathrm{D}$ position within the field; this is the foundation of ENB (35). Broadly, ENB begins like VBN: CT data is imported, the target is selected, and a navigation route is generated. This route can be reviewed manually as a form of VBN. Registration of an electromagnetic sensor (moving within a field around the patient's chest) to an imported scan allows sensor position to be displayed in relation to the patient's anatomy.

There are currently two approved ENB systems, which differ slightly in their workflow. The superDimension (Medtronic, USA) system consists of four key components: the location board, patient sensors, the disposable extended working channel (EWC), and the disposable locatable guide (LG). Planning can be done using any recent highquality CT chest. The location board is placed under the patient and generates the electromagnetic field. Patient sensors, placed on the chest, allow compensation for patient movement. The LG contains the electromagnetic sensor and fits within the EWC. To register the CT data to the ENB system, the EWC/LG is inserted through the bronchoscope and held at designated targets (e.g., main carina) or, in newer versions, passed through each lobar bronchus for automatic registration. The EWC is angulated, and thus functions as a steerable GS to selectively access bronchial branches along the navigation route. Once the target is reached, the EWC is locked in place and the LG removed to allow use of other instruments.

The SPiN System (Veran Medical Technologies, USA) consists of three key components: the vPads, the field generator, and tracked instruments. A set of inspiratory and expiratory CT scans must be acquired on the procedure day with the vPads (a set of 3 adhesive sensors) already secured on the patient's anterior chest. When the scans are imported into the planning software, markers in the vPads are used for automatic registration (hence the requirement for sameday imaging to reduce registration error). Inspiratory and expiratory scans are used for respiratory gating, with breathing detected by the vPads. During ENB, the field generator is placed directly over the patient and disposable instruments with embedded electromagnetic sensors are used.

Data on ENB performance is influenced on the use of adjunctive technologies, like VBN. Eberhardt and colleagues randomized 118 patients undergoing transbronchial nodule biopsy to RP-EBUS alone, superDimension alone, or superDimension plus RP-EBUS (36). Diagnostic yield was significantly better with the combined approach $(88 \%$, $\mathrm{P}=0.02$ ) compared to RP-EBUS (69\%) and ENB (59\%) (36). ENB performed significantly worse in the lower lobe (29\%, P=0.01), in keeping with CT-body divergence (36). Several groups subsequently evaluated conditional use of ENB. A retrospective study of 60 patients undergoing transbronchial biopsy of 10-60 mm lesions with RP-EBUS found that performing superDimension ENB after failed RP-EBUS visualization improved lesion localization (93\% versus $75 \%, \mathrm{P}=0.001$ ) (37). Lesions requiring $\mathrm{ENB}$ were generally smaller (22 versus $30 \mathrm{~mm}, \mathrm{P}<0.05$ ), less likely to have a bronchus sign $(33 \%$ versus $76 \%, \mathrm{P}<0.01)$, and more likely to be in the upper lobe $(\mathrm{P}<0.05)(37)$. However, ENB did not significantly improve diagnostic yield (50\% versus 
$43 \%, \mathrm{P}=0.125$ ) (37). A prospective study of 236 consecutive patients evaluated use of superDimension ENB after RPEBUS and 'VBN' (i.e., review of superDimension planning data) failure (38). The addition of ENB improved RPEBUS visualization from $77 \%$ to $85.3 \%$ (38). However, the improvement in diagnostic yield was marginal (54.7\% versus $58.4 \%$ ) (38). Lesions requiring ENB were again smaller (23.8 versus $19.1 \mathrm{~mm}, \mathrm{P}=0.006$ ) (38).

The inability to replicate the results of the Eberhardt trial may reflect the real-world experience with ENB. A 2014 meta-analysis calculated the pooled diagnostic yield of ENB to be $64.9 \%$ (39). On the lower end, a multicentre registry of transbronchial peripheral nodule biopsy reported the diagnostic yield of ENB alone to be $38.5 \%$, improving to $47.1 \%$ when coupled with RP-EBUS (40). On the higher end, the NAVIGATE trial, a large multicenter single-cohort study of 1,215 subjects who underwent SuperDimension ENB, reported a diagnostic yield of $72.9 \%$ on 1 year follow-up (41). Complete 24-month follow-up data is expected soon and may help clarify realistic expectations on the benefits of ENB.

There have been no head-to-head comparisons between the superDimension and SPiN System. CT-body divergence supports the value of the SPiN System's respiratory gating; however, a review of diagnostic yields across ENB studies do not reveal any obvious differences (35). Both systems continue to receive software updates that improve their performance and capabilities. For example, a recent update to the superDimension software introduced a feature for correcting CT-body divergence using intraoperative fluoroscopic tomosynthesis (42). A retrospective study comparing standard ENB ( $n=90)$ with tomosynthesis-corrected ENB ( $n=59)$ found diagnostic yield was significantly improved (54\% versus $79 \%, \mathrm{P}=0.0019$ ); the median registration correction was $12.2 \mathrm{~mm}$ (42).

Reduced patient movement through general anesthesia may also have benefit in reducing CT-body divergence. One meta-analysis found ENB diagnostic yield with general anesthesia outperformed intravenous sedation (69.2\% versus $57.5 \%, \mathrm{P}=0.02$ ) (39). However, there was significant heterogeneity between studies using general anesthesia and those using intravenous sedation. One retrospective study directly comparing general anesthesia $(n=62)$ and intravenous sedation $(\mathrm{n}=58)$ found no difference in diagnostic yield ( $70 \%$ versus $78 \%, \mathrm{P}=0.38$ ), but significantly reduced procedure time with intravenous sedation (58 versus 43 min, $\mathrm{P}=0.0005$ ) (43).

In summary, existing data supports the use of ENB in combination with other modalities, particularly RP-EBUS. Lesions benefiting from ENB are generally smaller $(\leq 20 \mathrm{~mm})$. However, the magnitude of ENB's benefit remains less clear. Improved RP-EBUS localization success with ENB suggests the system can improve access to the area of the nodule, but this does not necessarily translate to nodule access itself. This distinction may be irrelevant for procedures that do not require precise access, such as preoperative pleural dye marking. Discrepancy between localization and diagnostic yield may be multifactorial, such as shifting EWC position during RP-EBUS exchange, but CT-body divergence likely plays a significant role. Refinement in CT-body divergence mitigation strategies, such as enhanced respiratory gating or tomosynthesis-correction, may improve ENB's performance over time. A major limitation of ENB is cost, which includes both installation fees and disposable instruments. Thoughtful identification of cases most likely to benefit from ENB are critical to its cost-effective use.

\section{Bronchoscopy}

\section{$U T B$}

The outer diameter cut-off between conventional, thin, and UTB is not clearly defined, although $\leq 3 \mathrm{~mm}$ for UTB and $\geq 5 \mathrm{~mm}$ for conventional bronchoscopy is often quoted $(8,44,45)$. UTB's smaller outer diameter and increased range of motion improves maneuverability within the peripheral bronchial tree. This has historically come at the cost of reduced working channel size; however, this cost is reduced in newer generation UTB (MP-190F/290F, Olympus, Japan; $3 \mathrm{~mm}$ outer diameter, $1.7 \mathrm{~mm}$ working channel).

One non-inferiority trial randomized 305 patients with nodules $\leq 30 \mathrm{~mm}$ to UTB or thin bronchoscopy ( $4 \mathrm{~mm}$ outer diameter, $2 \mathrm{~mm}$ working channel) (46). All procedures were performed with Bf-NAVI and RP-EBUS; however, the thin bronchoscopy group used a GS (46). The noninferiority margin ( $-10 \%$ diagnostic yield) was met, with UTB outperforming thin bronchoscopy (75\% versus $61 \%$, $\mathrm{P}=0.008$ ) (46). Subgroup analysis revealed UTB performed better for nodules in the peripheral-third of the lung $(\mathrm{P}=0.002)$ and bronchus sign-positive lesions $(\mathrm{P}=0.001)(46)$. Consistent with these findings, UTB could reach more peripherally (median $5^{\text {th }}$ versus $4^{\text {th }}$ bronchial generation, $\mathrm{P}<0.001)$ (46). A potential criticism of this trial was that both groups used the same biopsy instruments, and thus the thin bronchoscopy group did not fully leverage the larger working channel (e.g., larger forceps, transbronchial 

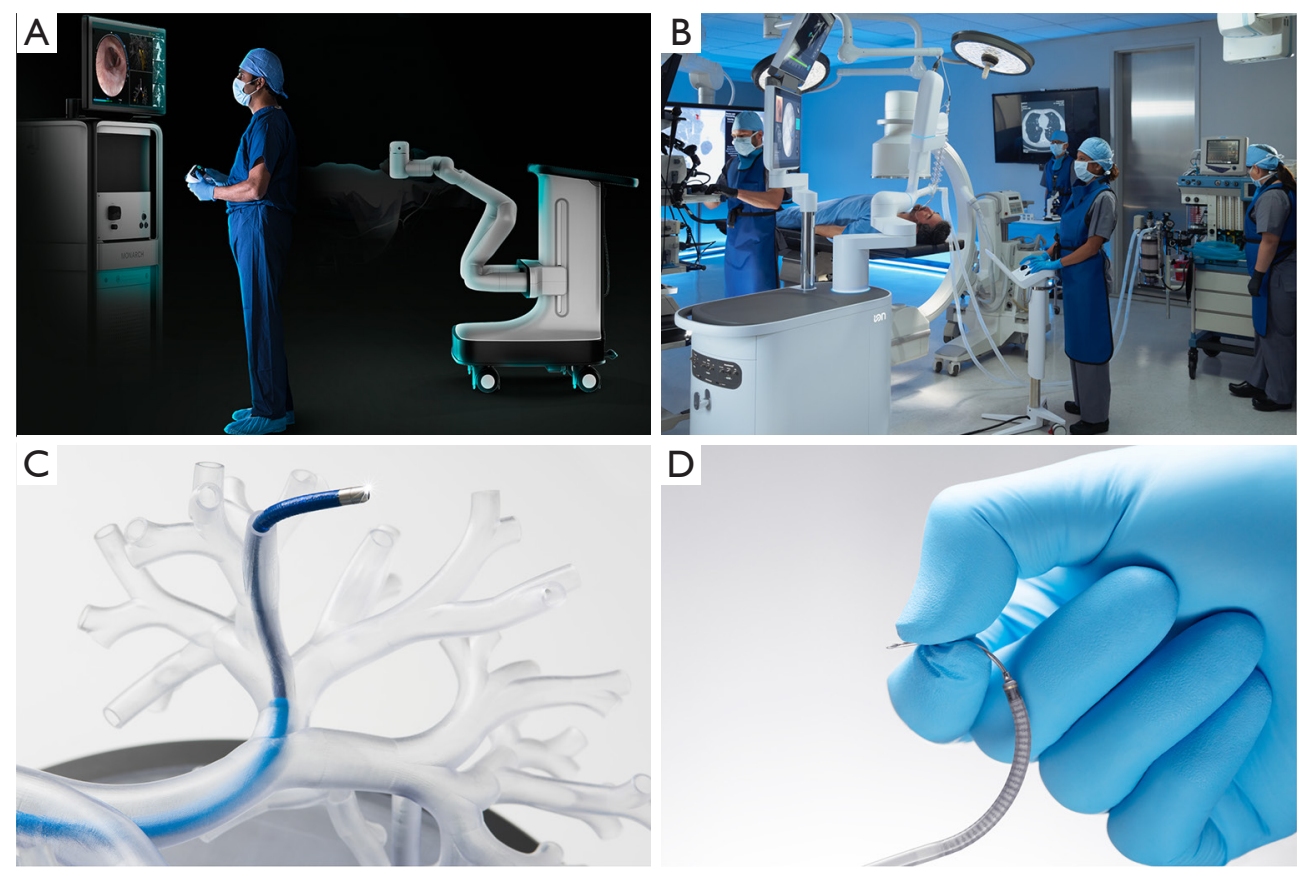

Figure 1 Robotic bronchoscopy platforms. The two currently available robotic bronchoscopy platforms are the Monarch ${ }^{\circledR}$ from Auris ${ }^{\mathrm{TM}}$ Health, Inc. (A) and the Ion ${ }^{\mathrm{TM}}$ from Intuitive Surgical ${ }^{\circledR}$, Inc. (B). The two systems differ in several key aspects. For example, the former is manipulated using a handheld controller (A), while the latter uses an upright console (B). The Monarch ${ }^{\circledR}$ employs a telescoping setup, whereby a larger catheter (light blue) can be parked before advancing a flexible inner section that contains the working channel (dark blue, C). The Ion ${ }^{\mathrm{TM}}$, by comparison, uses a single insertion tube design (D). Images (C2020 Auris ${ }^{\mathrm{TM}}$ Health, Inc. and Intuitive Surgical $^{\circledR}$, Inc. Reproduced with permission.

needle aspiration). As such, the group conducted a second randomized trial allocating 356 patients to either UTB or thin bronchoscopy without requiring GS use (47). The advantage of UTB was reaffirmed (70.1\% versus $58.7 \%$ diagnostic yield, $\mathrm{P}=0.027$ ) (47).

These trials support use of modern UTB for nodule access, particularly small peripheral nodules. Institutions regularly using VBN and/or RP-EBUS can easily integrate these devices into their current workflows. However, to our knowledge the working channel remains too small for existing ENB systems. This makes the choice between UTB and ENB mutually exclusive, and thus their relative performance will be of interest. Of note, diagnostic yields in both UTB randomized trials were similar to that reported in a large longitudinal ENB trial, NAVIGATE (41).

\section{Robotic bronchoscopy}

Fundamental features of bronchoscope design can contribute to difficulty accessing peripheral nodules.
Transmission of mechanical force from the bronchoscope handle to the tip can be limited by bend or loop formation in the insertion tube. The inability to provide consistent forward force can also contribute to tip slippage during bronchoscope or patient movement, resulting in loss of nodule access. Furthermore, accessing peripheral bronchi can require subtle movements that may be difficult to perform using rotation and flexion-extension alone, particularly if rotation results in compromised endoscopic visualization.

Robotic bronchoscopes aim to address these challenges by offering increased stability and maneuverability. Two platforms have regulatory approval: Monarch (Auris Health Inc., USA) and Ion (Intuitive Surgical Inc., USA) (Figure 1).

Monarch employs a 'telescoping' design that embeds an inner $4.4 \mathrm{~mm}$ scope (2.1 mm working channel) within a $6.0 \mathrm{~mm}$ outer sheath; the bronchoscope is operated using a handheld controller (48). The inner and outer components can be manipulated together or independently (48). 'Parking' the outer sheath can provide additional stability as the inner scope 
is advanced further ahead (49). Navigation is provided through integrated ENB capabilities (50). By comparison, Ion consists of a single $3.5 \mathrm{~mm}$ insertion tube with $2.0 \mathrm{~mm}$ working channel, operated via a console using a scroll wheel and track ball (51). Embedded within the device is a shape sensing fiber that monitors the scope's configuration in $3 \mathrm{D}$ space; this data can be used for registration with a preoperative CT scan to provide intraoperative navigation (52).

\section{Monarch (Auris Health)}

Monarch has shown potential performance advantages over flexible bronchoscopy in a series of cadaver studies. Despite its larger outer diameter, Monarch demonstrated improved peripheral reach compared to thin bronchoscopy $(4.2 \mathrm{~mm}$ outer diameter) in every lung segment of mechanically ventilated cadavers, as measured by increased mean bronchial generation count (range, 1-6) and increased mean insertion depth (range, 35.1-48.4 mm) (49). A follow-up study with randomly placed small peripheral pseudotumors found the diagnostic yield to be $94 \%$ when used with RPEBUS and ENB (48).

The first Monarch clinical experience was reported in 15 patients; RP-EBUS and the system's ENB capabilities were not used (53). Tissue was obtained in 14 cases without complication (53). A subsequent multicenter study in 165 patients reported $88.6 \%$ navigation success and a diagnostic yield of $69.1 \%$ to $77 \%$ (depending on classification of patients with insufficient follow-up data) when combined with ENB and RP-EBUS (54). The pneumothorax rate was $3.6 \%$, and airway bleeding requiring exchange with a standard bronchoscope for intervention occurred in $2.4 \%$ of cases (54).

\section{Ion (Intuitive Surgical)}

Comparison between UTB/RP-EBUS alone, superDimension alone, and Ion alone was performed in a cadaver model with randomly placed small pseudotumors (51). Participants deployed the biopsy needle when a nodule was localized by RP-EBUS or ENB/Ion navigation, and biopsy needle position was confirmed by CBCT (51). Nodule localization success was $65 \%$ with UTB/RP-EBUS, $85 \%$ with ENB, and $100 \%$ with Ion (51). Successful puncture was significantly greater with Ion (80\%) compared to ENB (45\%, $\mathrm{P}=0.022)$ and UTB/RPEBUS $(25 \%, \mathrm{P}<0.001)(51)$.

The first clinical report of Ion, in conjunction with RPEBUS, included 29 patients (52). Nodule access (defined by retrieval of tissue other than normal lung) was $96.6 \%$; diagnostic yield was $79.3 \%$ (52). No pneumothorax or airway injury occurred (52).

\section{Summary}

Experience with robotic bronchoscopy is preliminary but encouraging. Further studies will be required before the cost-effectiveness of a robotic bronchoscopy program, including differences between Monarch and Ion, can be estimated. Notably, all clinical trials have used general anesthesia, which increases the logistical burden of such procedures.

\section{Transparenchymal access}

Transbronchial techniques are constricted by the bronchial anatomy, with limited access to nodules not immediately adjacent to the bronchial wall. This is reflected by multiple studies demonstrating an association between diagnostic yield and the presence of a bronchus sign on CT or concentric visualization on RP-EBUS (8). Transparenchymal systems integrate navigation technologies and tunneling instruments to access nodules not otherwise amenable for transbronchial approaches.

The Archimedes Virtual Bronchoscopy Navigation System (Broncus Medical Inc., USA), also referred to as bronchoscopic transparenchymal nodule access (BTPNA), employs a LungPoint VBN-like foundation. During planning, the target is marked, and the system identifies a point of entry (POE) along the bronchial wall that provides a direct route without traversing vessels (55). This information is used to generate a final BTPNA-VBN plan for navigating to the POE. BTPNA is performed by puncturing the POE with a coring needle, dilating using a balloon dilator, and then advancing a GS with blunt dissecting stylet (55). Guidance then transitions from VBN to augmented fluoroscopy, displaying a $2 \mathrm{D}$ projection of the navigation plan and nodule location (55). Once the target is reached the stylet is removed, and instruments can be placed through the sheath.

CrossCountry (Medtronic, USA), also referred to as the transbronchial access tool (TBAT), employs superDimension ENB. During planning, the target nodule, desired exit point, and danger zones (e.g., vessels) are marked (56). A plan is then generated that can be modified to ensure tunneling through the danger zone is avoided (56). TBAT begins as ENB with navigation to the exit point, at which point the LG is exchanged for a puncture wire (56). After puncturing the bronchial wall, a Seldinger technique is used (56). A dilator is fed over the wire to tunnel to the nodule (56). Once in position, the EWC is advanced over the dilator, the dilator is removed, and instruments can be passed through the EWC (56). 


\section{Archimedes (bronchoscopic transparenchymal nodule access)}

A BTPNA-and-resect trial was performed in 12 patients, with a diagnostic yield of $83 \%$ (55). The two non-diagnostic cases were in the apical left upper lobe (55). BTPNA could not be performed in those cases (55). A subsequent series of BTPNA alone in 6 patients reported procedure success (including diagnostic tissue) in 5 cases; the 1 failed case was due to an error generating the augmented fluoroscopy image (57). Two cases developed a pneumothorax, with one requiring chest drain; both cases had nodules close to the pleura (57).

\section{CrossCountry (transbronchial access tool)}

The first series of TBAT included 3 patients with nodules inaccessible by conventional ENB (58). CBCT and/or fluoroscopy were used in all cases (58). There were no complications, although one case was non-diagnostic (58). A subsequent series of 12 patients with TBAT and CBCT reported a procedural success rate of $75 \%$ with an overall diagnostic yield of $66 \%$ (56). One pneumothorax occurred, requiring chest drain (56). Failed TBAT access occurred in areas requiring sharply angled approaches (56).

\section{Summary}

BTPNA/TBAT are feasible. However, additional safety and performance information is needed. The potential consequences of CT-body divergence on vessel (rather than nodule) position remains to be seen, with the incumbent risks of inadvertent vessel puncture. Updates to VBN and ENB software may simplify the planning process for BTPNA/TBAT. General anesthesia will likely be required, given the risks associated with uncontrolled parenchymal dissection during unexpected movement. BTPNA/TBAT may also have some difficulties in areas of high angulation. Placement of instruments in the EWC reportedly caused unintended straightening that resulted in misalignment with the planned puncture site (56). The tip stability and maneuverability of robotic platforms may ultimately be one strategy to address these challenging regions.

\section{Conclusions}

A variety of technologies are available to facilitate nodule access. Many, such as VBN, ENB, and UTB, are showing continued maturation and evolution. Others, such as robotic bronchoscopy and transparenchymal access, are in the early phases of their evaluation. A common thread in review of these systems is that their performance is optimized through thoughtful combination with other relevant technologies. Reliance on any single platform alone is unlikely to realize significant benefits for nodule access. Navigation platforms by their nature rely on preoperative imaging and planning procedures prior to use. Patients that may require navigation must therefore be identified before their scheduled bronchoscopy. Like much in medicine, there continues to be a need for large multicentre randomized trials both for nascent (e.g., robotic bronchoscopy) and evolving (e.g., ENB) technologies to understand the costeffectiveness of these increasingly complex systems.

The literature suggests, as expected, that these technologies offer most benefit for lesions that are poorly accessed by conventional bronchoscopy: small $(\leq 20 \mathrm{~mm})$ nodules in the periphery (outer one-third lung field). Current trends indicate the future of bronchoscopy is smaller, more maneuverable bronchoscopes complemented by navigation systems that can correct for CT-body divergence. However, routine upfront use of advanced technologies in all bronchoscopic procedures is unlikely to be cost-effective. Rather, careful review of the patient's CT scan, familiarity with RP-EBUS, and availability of fluoroscopy remain invaluable, serving as a foundation upon which additional technologies should be applied.

\section{Acknowledgments}

The authors would like to thank Judy McConnell (Toronto General Hospital) for her assistance with laboratory management.

Funding: KY is supported by the William Coco Chair in Surgical Innovation for Lung Cancer. AG is supported by a Canadian Institutes of Health Research Frederick Banting and Charles Best Doctoral Canada Graduate Scholarship (FRN 170883) and a University of Toronto Temerty Faculty of Medicine Hold'em for Life Oncology Clinician Scientist Award.

\section{Footnote}

Provenance and Peer Review: This article was commissioned by the Guest Editors (Scott Swanson, Daniel Dolan) for the series "How to Evaluate, Diagnose and Treat Small Lung Nodules" published in Current Challenges in Thoracic Surgery. The article has undergone external peer review. 
Reporting Checklist: The authors have completed the Narrative Review reporting checklist. Available at https:// ccts.amegroups.com/article/view/10.21037/ccts-20-122/rc Peer Review File: Available at https://ccts.amegroups.com/ article/view/10.21037/ccts-20-122/prf

Conflicts of Interest: All authors have completed the ICMJE uniform disclosure form (available at https://ccts. amegroups.com/article/view/10.21037/ccts-20-122/coif). The series "How to Evaluate, Diagnose and Treat Small Lung Nodules" was commissioned by the editorial office without any funding or sponsorship. AG reports grants from the Canadian Institutes of Health Research, and grants from the University of Toronto Temerty Faculty of Medicine/Hold'em for Life Charity Challenge, during the conduct of the study. KY reports grants and personal fees from Olympus Corporation, personal fees from Intuitive Surgical, personal fees from Medtronic, grants and personal fees from Johnson \& Johnson, and grants from Siemens, outside the submitted work. In addition, KY is on the Advisory Board of Auris Heath Inc and Concordia Healthcare, outside the submitted work. The authors have no other conflicts of interest to declare.

Ethical Statement: The authors are accountable for all aspects of the work in ensuring that questions related to the accuracy or integrity of any part of the work are appropriately investigated and resolved.

Open Access Statement: This is an Open Access article distributed in accordance with the Creative Commons Attribution-NonCommercial-NoDerivs 4.0 International License (CC BY-NC-ND 4.0), which permits the noncommercial replication and distribution of the article with the strict proviso that no changes or edits are made and the original work is properly cited (including links to both the formal publication through the relevant DOI and the license). See: https://creativecommons.org/licenses/by-nc-nd/4.0/.

\section{References}

1. Aberle DR, Adams AM, Berg CD, et al. Reduced LungCancer Mortality with Low-Dose Computed Tomographic Screening. N Engl J Med 2011;365:395-409.

2. de Koning HJ, Van Der Aalst CM, De Jong PA, et al. Reduced lung-cancer mortality with volume CT screening in a randomized trial. N Engl J Med 2020;382:503-13.

3. Shirvani SM, Jiang J, Chang JY, et al. Lobectomy, sublobar resection, and stereotactic ablative radiotherapy for earlystage non-small cell lung cancers in the elderly. JAMA Surg 2014;149:1244-53.

4. Han Y, Kim HJ, Kong KA, et al. Diagnosis of small pulmonary lesions by transbronchial lung biopsy with radial endobronchial ultrasound and virtual bronchoscopic navigation versus CT-guided transthoracic needle biopsy: A systematic review and meta-analysis. PLoS One 2018;13:e0191590.

5. Wang Memoli JS, Nietert PJ, Silvestri GA. Metaanalysis of guided bronchoscopy for the evaluation of the pulmonary nodule. Chest 2012;142:385-93.

6. Rivera MP, Mehta AC, Wahidi MM. Establishing the diagnosis of lung cancer: Diagnosis and management of lung cancer, 3rd ed: American college of chest physicians evidence-based clinical practice guidelines. Chest 2013;143:e142S-e165S.

7. Tsukada H. EBUS and EM guided transthoracic needle aspiration for peripheral small lung nodules not amenable to radiographic tissue sampling. Curr Challenges Thorac Surg 2021. doi: 10.21037/ccts-20-106.

8. Ishiwata T, Gregor A, Inage T, et al. Advances in interventional diagnostic bronchoscopy for peripheral pulmonary lesions. Expert Rev Respir Med 2019;13:885-97.

9. Carlson SK, Bender CE, Classic KL, et al. Benefits and safety of CT fluoroscopy in interventional radiologic procedures. Radiology 2001;219:515-20.

10. Tsushima K, Sone S, Hanaoka T, et al. Comparison of bronchoscopic diagnosis for peripheral pulmonary nodule under fluoroscopic guidance with CT guidance. Respir Med 2006;100:737-45.

11. Ost D, Shah R, Anasco E, et al. A randomized trial of CT fluoroscopic-guided bronchoscopy vs conventional bronchoscopy in patients with suspected lung cancer. Chest 2008;134:507-13.

12. Ng CSH, Chu CM, Lo CK, et al. Hybrid operating room Dyna-computed tomography combined imageguided electromagnetic navigation bronchoscopy dye marking and hookwire localization video-assisted thoracic surgery metastasectomy. Interact Cardiovasc Thorac Surg 2018;26:338-40.

13. Casal RF, Sarkiss M, Jones AK, et al. Cone beam computed tomography-guided thin/ultrathin bronchoscopy for diagnosis of peripheral lung nodules: A prospective pilot study. J Thorac Dis 2018;10:6950-9.

14. Yang SM, Yu KL, Lin KH, et al. Real-time augmented fluoroscopy-guided lung marking for thoracoscopic 
resection of small pulmonary nodules. Surg Endosc 2020;34:477-84.

15. Pritchett MA, Schampaert S, De Groot JAH, et al. ConeBeam CT with Augmented Fluoroscopy Combined with Electromagnetic Navigation Bronchoscopy for Biopsy of Pulmonary Nodules. J Bronchology Interv Pulmonol 2018;25:274-82.

16. Krimsky WS, Pritchett MA, Lau KKW. Towards an optimization of bronchoscopic approaches to the diagnosis and treatment of the pulmonary nodules: A review. J Thorac Dis 2018;10:S1637-44.

17. Hogarth D, Bhadra K, Whitten P, et al. A novel endobronchial fluoroscopic navigation and localization system: a summary of a multicenter LungVision trial. Chest 2018;154:880A-882A.

18. Semaan RW, Lee HJ, Feller-Kopman D, et al. Sameday computed tomographic chest imaging for pulmonary nodule targeting with electromagnetic navigation bronchoscopy may decrease unnecessary procedures. Ann Am Thorac Soc 2016;13:2223-8.

19. Chen A, Pastis N, Furukawa B, et al. The effect of respiratory motion on pulmonary nodule location during electromagnetic navigation bronchoscopy. Chest 2015;147:1275-81.

20. Leira HO, Langø T, Sorger H, et al. Bronchoscopeinduced Displacement of Lung Targets. J Bronchology Interv Pulmonol 2013;20:206-12.

21. Asano F, Matsuno Y, Shinagawa N, et al. A virtual bronchoscopic navigation system for pulmonary peripheral lesions. Chest 2006;130:559-66.

22. Shinagawa N, Yamazaki K, Onodera Y, et al. Virtual bronchoscopic navigation system shortens the examination time-Feasibility study of virtual bronchoscopic navigation system. Lung Cancer 2007;56:201-6.

23. Sano A, Tsuchiya T. Virtual bronchoscopy using OsiriX. J Bronchol Interv Pulmonol 2014;21:113-6.

24. Miyake K, Morimura O, Inoue T, et al. The Direct Oblique Method. J Bronchology Interv Pulmonol 2018;25:305-14.

25. Miyoshi S, Isobe K, Shimizu H, et al. The utility of virtual bronchoscopy using a computed tomography workstation for conducting conventional bronchoscopy: A retrospective analysis of clinical practice. Respiration 2019;97:52-9.

26. Asano F, Eberhardt R, Herth FJF. Virtual bronchoscopic navigation for peripheral pulmonary lesions. Respiration 2014;88:430-40.

27. Eberhardt R, Kahn N, Gompelmann D, et al. LungPoint - A new approach to peripheral lesions. J Thorac Oncol
2010;5:1559-63.

28. Oshige M, Shirakawa T, Nakamura M, et al. Clinical application of virtual bronchoscopic navigation system for peripheral lung lesions. J Bronchology Interv Pulmonol 2011;18:196-202.

29. Bo L, Li C, Pan L, et al. Diagnosing a solitary pulmonary nodule using multiple bronchoscopic guided technologies: A prospective randomized study. Lung Cancer 2019;129:48-54.

30. Ishida T, Asano F, Yamazaki K, et al. Virtual bronchoscopic navigation combined with endobronchial ultrasound to diagnose small peripheral pulmonary lesions: A randomised trial. Thorax 2011;66:1072-7.

31. Xu C, Yuan Q, Wang Y, et al. Usefulness of virtual bronchoscopic navigation combined with endobronchial ultrasound guided transbronchial lung biopsy for solitary pulmonary nodules. Medicine (Baltimore) 2019;98:e14248.

32. Asano F, Shinagawa N, Ishida T, et al. Virtual bronchoscopic navigation combined with ultrathin bronchoscopy a randomized clinical trial. Am J Respir Crit Care Med 2013;188:327-33.

33. Diez-Ferrer M, Morales A, Tebé C, et al. Ultrathin Bronchoscopy with and without Virtual Bronchoscopic Navigation: Influence of Segmentation on Diagnostic Yield. Respiration 2019;97:252-8.

34. Kato A, Yasuo M, Tokoro Y, et al. Virtual bronchoscopic navigation as an aid to CT-guided transbronchial biopsy improves the diagnostic yield for small peripheral pulmonary lesions. Respirology 2018;23:1049-54.

35. Mehta AC, Hood KL, Schwarz Y, et al. The Evolutional History of Electromagnetic Navigation Bronchoscopy: State of the Art. Chest 2018;154:935-47.

36. Eberhardt R, Anantham D, Ernst A, et al. Multimodality bronchoscopic diagnosis of peripheral lung lesions: A randomized controlled trial. Am J Respir Crit Care Med 2007;176:36-41.

37. Chee A, Stather DR, Maceachern P, et al. Diagnostic utility of peripheral endobronchial ultrasound with electromagnetic navigation bronchoscopy in peripheral lung nodules. Respirology 2013;18:784-9.

38. Steinfort DP, Bonney A, See K, et al. Sequential multimodality bronchoscopic investigation of peripheral pulmonary lesions. Eur Respir J 2016;47:607-14.

39. Gex G, Pralong JA, Combescure C, et al. Diagnostic yield and safety of electromagnetic navigation bronchoscopy for lung nodules: A systematic review and meta-analysis. Respiration 2014;87:165-76.

40. Ost DE, Ernst A, Lei X, et al. Diagnostic yield and 
complications of bronchoscopy for peripheral lung lesions: Results of the AQuIRE registry. Am J Respir Crit Care Med 2016;193:68-77.

41. Folch EE, Pritchett MA, Nead MA, et al. Electromagnetic Navigation Bronchoscopy for Peripheral Pulmonary Lesions: One-Year Results of the Prospective, Multicenter NAVIGATE Study. J Thorac Oncol 2019;14:445-58.

42. Aboudara M, Roller L, Rickman O, et al. Improved diagnostic yield for lung nodules with digital tomosynthesis-corrected navigational bronchoscopy: Initial experience with a novel adjunct. Respirology 2020;25:206-13.

43. Bowling MR, Kohan MW, Walker P, et al. The Effect of General Anesthesia Versus Intravenous Sedation on Diagnostic Yield and Success in Electromagnetic Navigation Bronchoscopy. J Bronchology Interv Pulmonol 2015;22:5-13.

44. Shinagawa N. A review of existing and new methods of bronchoscopic diagnosis of lung cancer. Respir Investig 2019;57:3-8.

45. Asano F. Advanced bronchoscopy for the diagnosis of peripheral pulmonary lesions. Respir Investig 2016;54:224-9.

46. Oki M, Saka H, Ando M, et al. Ultrathin bronchoscopy with multimodal devices for peripheral pulmonary lesions: A randomized trial. Am J Respir Crit Care Med 2015;192:468-76.

47. Oki M, Saka H, Asano F, et al. Use of an Ultrathin vs Thin Bronchoscope for Peripheral Pulmonary Lesions: A Randomized Trial. Chest 2019;156:954-64.

48. Chen AC, Pastis NJ, Machuzak MS, et al. Accuracy of a Robotic Endoscopic System in Cadaver Models with Simulated Tumor Targets: ACCESS Study. Respiration 2020;99:56-61.

49. Chen AC, Gillespie CT. Robotic Endoscopic Airway Challenge: REACH Assessment. Ann Thorac Surg 2018;106:293-7.

doi: $10.21037 /$ ccts-20-122

Cite this article as: Gregor A, Ishiwata T, Inage T, Yasufuku K. Narrative review-how to access nodules: role of new technology including navi- and robo-bronchoscopy. Curr Chall Thorac Surg 2022;4:34.
50. Murgu SD. Robotic assisted-bronchoscopy: Technical tips and lessons learned from the initial experience with sampling peripheral lung lesions. BMC Pulm Med 2019;19:89.

51. Yarmus L, Akulian J, Wahidi M, et al. A Prospective Randomized Comparative Study of Three Guided Bronchoscopic Approaches for Investigating Pulmonary Nodules: The PRECISION-1 Study. Chest 2020;157:694701.

52. Fielding DIK, Bashirzadeh F, Son JH, et al. First Human Use of a New Robotic-Assisted Fiber Optic Sensing Navigation System for Small Peripheral Pulmonary Nodules. Respiration 2019;98:142-50.

53. Rojas-Solano JR, Ugalde-Gamboa L, MacHuzak M. Robotic Bronchoscopy for Diagnosis of Suspected Lung Cancer: A Feasibility Study. J Bronchology Interv Pulmonol 2018;25:168-75.

54. Chaddha U, Kovacs SP, Manley C, et al. Robot-assisted bronchoscopy for pulmonary lesion diagnosis: Results from the initial multicenter experience. BMC Pulm Med 2019;19:243.

55. Herth FJF, Eberhardt R, Sterman D, et al. Bronchoscopic transparenchymal nodule access (BTPNA): First in human trial of a novel procedure for sampling solitary pulmonary nodules. Thorax 2015;70:326-32.

56. Bowling MR, Brown C, Anciano CJ. Feasibility and Safety of the Transbronchial Access Tool for Peripheral Pulmonary Nodule and Mass. Ann Thorac Surg 2017;104:443-9.

57. Harzheim D, Sterman D, Shah PL, et al. Bronchoscopic Transparenchymal Nodule Access: Feasibility and Safety in an Endoscopic Unit. Respiration 2016;91:302-6.

58. Anciano C, Brown C, Bowling M. Going off Road: The First Case Reports of the Use of the Transbronchial Access Tool with Electromagnetic Navigational Bronchoscopy. J Bronchology Interv Pulmonol 2017;24:253-6. 